\section{Watermelon esophagus: a new visual paradigm}

\author{
Kevin Cowley, ${ }^{1}$ H. Whitney Jennings, ${ }^{2}$ \\ Chad Burski, ${ }^{2}$ Michael Passarella ${ }^{2}$ \\ ${ }^{1}$ Tinsley Harrison Internal Medicine \\ Residency Program, and ${ }^{2}$ Department of \\ Gastroenterology and Hepatology, \\ University of Alabama at Birmingham, \\ AL, USA
}

\begin{abstract}
A 35-year-old male with no prior medical history presented for evaluation of multi-year history of dysphagia to both solids and liquids. He underwent esophagogastroduodenoscopy revealing linear furrows with ring-like esophagus. Pathology of biopsies showed findings consistent with eosinophilic esophagitis (EoE). This is a newly recognized disease entity with a rapidly increasing prevalence that cannot be entirely accounted for by the increasing awareness in medicine. All patients with clinical suspicion of EoE should undergo esophageal biopsies regardless of endoscopic findings, as normal endoscopy can be seen in up to $17 \%$ of cases. Numerous non-specific endoscopic findings have been described, including trachealization, felinization, linear furrows, crepe paper mucosa, and longitudinal shearing. We present a unique and neverbefore seen image of EoE that accentuates the findings that may be seen endoscopically.
\end{abstract}

\section{Case Report}

A 35-year-old male with no prior medical history presented for evaluation of multi-year history of dysphagia to both solids and liquids. Laboratory data and modified barium swallow study were unrevealing of a cause of his dysphagia. After failure of an empiric eight-week trial of proton pump inhibitor therapy, he underwent esophagogastroduodenoscopy revealing linear furrows with ring-like esophagus. After biopsies were taken, mild bleeding accentuated the linear furrows, creating a watermelon esophagus appearance (Figure 1). Pathology of biopsies showed findings consistent with eosinophilic esophagitis.

\section{Discussion and Conclusions}

Eosinophilic esophagitis (EoE) is a primary clinico-pathologic disorder of the esophagus that is defined by clinical symptoms of esophageal dysfunction in addition to $\geq 15$ eosinopils/high-power field on esophageal mucuosal biopsy specimens. ${ }^{1}$ EoE is a newly recognized disease entity with a rapidly increasing prevalence that cannot be entirely accounted for by the increasing awareness in medicine. ${ }^{1} \mathrm{~A}$ high clinical suspicion is needed to make a diagnosis given the non-specific presenting symptoms, which may include dysphagia, food impaction, food aversion, heartburn, and chest pain. ${ }^{2}$ All patients with clinical suspicion of EoE should undergo esophageal

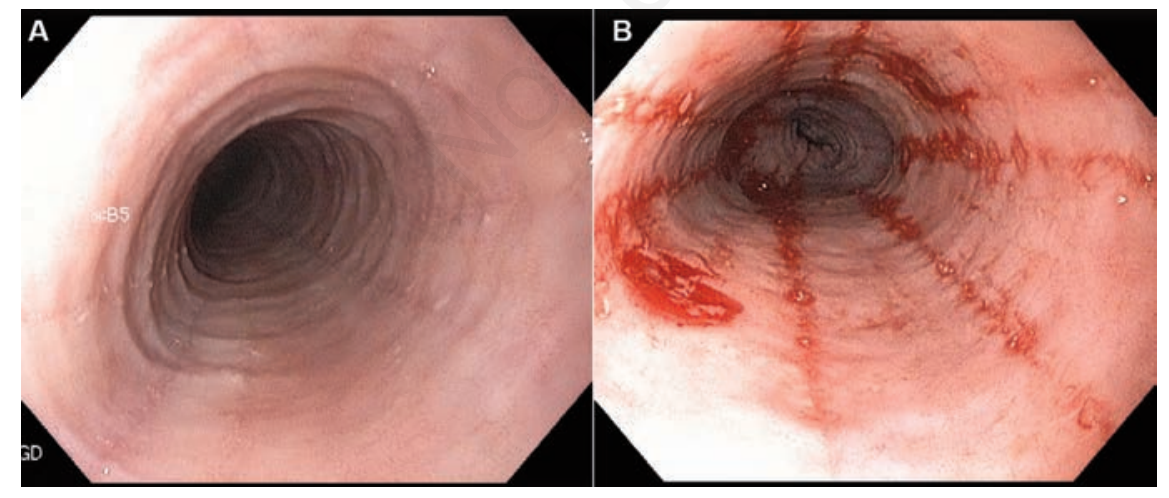

Figure 1. A) Trachealization of the esophagus with subtle linear furrows prior to biopsies; B) Esophagus with watermelon appearance after mid-esophageal biopsies.
Correspondence: Kevin Cowley, Tinsley Harrison Internal Medicine Residency Program, University of Alabama at Birmingham, 1720 2nd Ave., South BDB 327, Birmingham, AL 35294, USA.

Tel.: + 1.205.934.2490.

E-mail: kcowley@uabmc.edu

Key words: Eosinophilic esophagitis; endoscopy; esophagus.

Contributions: $\mathrm{KC}$ is the article guarantor and prepared the manuscript;. HWJ, CB, MP edited and approved the manuscript.

Conflict of interest: the authors declare no potential conflict of interest.

Received for publication: 13 July 2015.

Accepted for publication: 10 November 2015.

This work is licensed under a Creative Commons Attribution NonCommercial 3.0 License (CC BYNC 3.0).

(C) Copyright K. Cowley et al., 2015

Licensee PAGEPress, Italy

Gastroenterology Insights 2015; 6:6111

doi:10.4081/gi.2015.6111

biopsies regardless of endoscopic findings, as normal endoscopy can be seen in up to $17 \%$ of cases. ${ }^{3}$ Numerous non-specific endoscopic findings have been described, including trachealization, felinization, linear furrows, crepe paper mucosa, and longitudinal shearing. ${ }^{1} \mathrm{We}$ present a unique and never-before seen image of EoE that accentuates the findings that may be seen endoscopically.

\title{
References
}

1. Furuta GT, Liacouras CA, Collins MH, et al. Eosinophilic esophagitis in children and adults: a systematic review and consensus recommendations for diagnosis and treatment. Gastroenterology 2007;133:1342-63.

2. Carr S, Watson W. Eosinophilic esophagitis. Allergy Asthma Clin Immunol 2011;7:S8.

3 Kim HP, Vance RB, Shaheen NJ, Dellon ES. The prevalence and diagnostic utility of endoscopic features of eosinophilic esophagitis: a meta-analysis. Clin Gastroenterol Hepatol 2012;10:e5. 